\title{
The use of student narratives as research
}

\section{substrates}

\section{DESIGN INSIGHT}

\author{
Moyez Jiwa ${ }^{1}$, Catherine Krejany ${ }^{1}$, Elena Angelides ${ }^{2}$, Matthew Brown ${ }^{2}$, Rose Clarke 2 , Pia \\ Courtley², Julie Dao², Haissam Faraj², Meera Ghandi², Mitchell Gordon², Yi Zhao Huang², \\ Christian La Spina², Lauren McDermott², Nina Murphy², Marie Rattenbury², Alexander \\ Robertson², Henry Ruehland², William Thomas², Suzannah Bownes², Charlotte Krones², \\ Robert Wells², Gabriela Sousa Rocha²
}

1. Melbourne Clinical School, The University of Notre Dame Australia, Werribee, VIC, Australia

2. School of Medicine, Sydney, The University of Notre Dame Australia, Sydney, NSW, Australia

To Cite: Jiwa M, Krejany C, Brown M, Clarke R, Courtley P, Dao J, Faraj H, Ghandi M, Gordon M, Huang YZ, La Spina C, McDermott L, Murphy N, Rattenbury M, Robertson A, Ruehland H, Thomas W, Bownes S, Krones S, Krones C, Wells R, Rocha GS. The use of student narratives as research substrates. JHD. 2019;4(3):194-204. https://doi.org/10.21853/JHD.2019.86

\section{Corresponding Author:}

Moyez Jiwa

Melbourne Clinical School, School of Medicine

Sydney, University of Notre Dame Australia 300

Princes Hwy, Werribee, VIC 3030

moyez.jiwa@nd.edu.au

\section{Copyright:}

(C) 2019 The Authors. Published by Archetype Health Pty Ltd. This is an open access article under the CC BY-NC-ND 4.0 license.

\begin{abstract}
SUMMARY
Supervising medical students to develop a research project can be challenging Unless a student has prior experience in research, they may struggle to identify a research question that can be addressed by a novice researcher in a time-limited project. At the same time, when a project spans years of the degree, a student who has been offered an "off-the-shelf" idea may lose interest in the project and the assignment can become burdensome. We describe how a cohort of medical students were encouraged to focus their project ideas on a story of someone whose medical problem was of interest to them. Most students opted to conduct a literature review based on their "story". Here we present their stories and describe how they were led to this point in the process.
\end{abstract}

\section{Key Words}

medical education; research; medical students; medical schools; narrative medicine

\section{INTRODUCTION}

Many medical schools require undergraduate students to participate in research. The challenge when there is a large number of students in a cohort is to offer new projects every year that maintain students' interest for the duration of the assignment but also meet the program requirements. At the Sydney School of Medicine, University of Notre Dame Australia, students are required to lead a research project over four years as part of the requirements of their postgraduate degree. The project is assessed on the basis of a presentation at a research symposium, a poster, and a thesis submitted in the final year of a four-year program. In this paper, we describe the process of developing ideas for primary care and prevention stream after three meetings scheduled at three-week intervals with each student. The outcome in each case was a research question. Narrative medicine has a long established tradition in healthcare. As Fioretti et al. stated:

On the one hand medicine needs to be focused on scientifically-rigorous trials and to follow specific protocols, on the other hand the final aim of medical practice is always related to what a patient feels, what they perceive they feel and above all, what they say they feel. What scholars have pointed out is that listening to the patient's story is a tool to enrich not only the knowledge of their physical and psychological condition, but also to offer information with which to formulate the diagnosis. Thus, physicians and health staff need in their daily practice would seem to be to adopt a 'Narrative Evidence-Based Medicine'. ${ }^{1}$

In this process we deploy stories not to educate but to pique the interest and sustain effort in a four-year research project conducted by many who are novices to research. The "bottom-line" advice offered by one group of commentators in regard to helping medical students with research was: "For the student, pick a supervisor who is a timely finisher, and choose 
a project you like (both the topic and the "nuts and bolts" of it). For the supervisor, make sure the student wants to do the project and stress the importance of perseverance through the problems to get to the fun parts."

As this project starts in the first year, and is often most demanding at a time when students are immersed in their clinical training, we were keen to offer and implement the advice offered by Detsky and Detsky:

First, a commitment to do a research project must be made. Balancing medical school and a social life can be a challenge, and adding another activity will make considerable demands on your time. If you decide to do a project, acknowledge that this is something you are willing to carry through to completion, which may take years. Taking on a project that becomes more complex or demanding than one imagines is an error common among first-time researchers. If a smaller, less ambitious project presents itself, that may be a more appropriate first attempt at research. ${ }^{2}$

\section{SUMMARY}

Research projects were developed after three 20-minute face-to-face meetings with each of 20 students and two experienced researchers within the theme of "primary care and prevention". Students were invited to focus on as many details of the person whose medical problem had interested them. This could be a friend, relative, or acquaintance, or someone they had read about, met on the wards, or heard about from another source. The person had to be described in sufficient detail to outline their problem but most especially the context in which they were experiencing this problem. At the same time, the students were reminded that the person's privacy should not be breached and no identifying information was to be included in the story. In the course of the meetings with the supervisors the students were encouraged to add more details and to articulate clearly what aspect of the person's care was of most interest to them. This might have been the pathway to diagnosis, the risk factors at play, the help-seeking behavior, the role of the healthcare professionals in supporting the person, or the details of the treatment of the condition. The subjects of the story were from a community setting/primary care, and the research questions related to some aspect of the case that was relevant in that setting.

The students were invited to consider how the story of Joe was drafted in a paper in this journal and to review a video offering guidance on where to begin with the process. ${ }^{3,4}$ The story was expected to detail who (pseudonym), why, when, how, where, and what was interesting in the story of the person they had chosen to inspire their project. The team then worked with each individual student or team of two students to identify which aspect of the story was of most interest and to refine the research question. The student was then invited to find a paper from an informal review of the literature online that would be typical of the studies that they might review as part of their thesis.

\section{LESSONS LEARNED}

The process of generating narratives with a personal interest as a basis for research was an effective way to stimulate research ideas in a cohort of students who were mostly new to research. The series of stories offered by the students is outlined in the appendix. It was possible to progress from when the students had no topic for their project to where 20 students have now embarked on research. Most are systematic reviews of the literature, which can be completed in a library. Four key elements were identified that facilitated the process:

1. Each story reflects the student's background. Many are healthcare professionals by training but the group also includes many others who did not complete a science-based undergraduate degree. Some had conducted research previously but many were novices in research. The personalisation of the research context facilitated engagement with the research process regardless of previous research experience.

2. Short timeframe. This process was time efficient insofar as it led students to a topic that was meaningful to them 
Health Design

in three 20-minute sessions over a three-month period. Maintaining the momentum with regular four weekly meetings assisted focus and progress.

3. Facilitation of sessions by experienced researchers, including a practicing clinician, was vital to the process and enabled effective scoping and contextualizing of ideas.

4. Allow for flexibility within the timeframe. It was important to acknowledge that some students arrived at their "story" later in the meetings than others. Some students had not clearly articulated their story until at least the second meeting.

We found that this approach was extremely time efficient for both student and supervisors, all of whom often face competing demands. The approach also delivered a one-to-one mentoring experience within this short timeframe. The personal connection to the research narrative promoted student focus and engagement in research.

\section{DESIGN INSIGHT}

This paper outlines a process to help medical students develop achievable research projects that are developed from the stories of people that they know. The benefits of this approach include that the process of developing the research question encourages students to consider the link between research, practice, and evidence. It has practical advantages of developing research projects that do not require ethics approval, funding, recruitment, reliance on external agencies, or many of the other areas that can derail a research project timeline or plan. Not all medical students see research as a priority in their training-linking to clinical questions can encourage students to see the positives of being involved in research.

It is relevant to consider the learning objectives of the requirement to do a research project. Whilst systematic reviews are an important part of the research literature, they do rely on the quality of the research reviewed to be able to answer the question. Clinician researchers are uniquely placed to identify relevant research questions and be involved in developing and performing studies to answer those questions. Ensuring a significant number of medical students have the opportunity to develop a wide range of research skills is an important part of encouraging and supporting the next generation of clinician researchers. However, all doctors need a good understanding of how to find and assess relevant evidence-based information to guide their patient care.

Dr. Carolyn O'Shea, MBBS, MMed, FRACGP

General Practitioner

Greensborough, Vic, Australia

\section{REFERENCES}

1. Fioretti C, Mazzocco K, Riva S, et al. Research studies on patients' illness experience using the Narrative Medicine approach: a systematic review. BMJ Open. 2016 Jul 14;6(7):e011220. doi: 10.1136/bmjopen-2016-011220.

2. Detsky ME, Detsky AS. Encouraging medical students to do research and write papers. CMAJ. 2007 Jun 5;176(12):1719-21.

3. Krejany C, Jiwa M. Finding Joe: Patient-directed goal setting on the journey to therapeutic targets in diabetes care. JHD. 2019;4(1):168-171. doi: 10.21853/JHD.2018.80

4. The Art of Doctoring. MD video.[Internet] [2019 Jan 23]. [Accessed 2019 Jun 26]. Available from: https://www.youtube.com/watch?v=WTYi2_Yf8sk

\section{PEER REVIEW}

Not commissioned. Externally peer reviewed.

\section{CONFLICTS OF INTEREST}

The authors declare that they have no competing interests. 
FUNDING

None

ETHICS COMMITTEE APPROVAL

Not applicable. 


\section{APPENDIX}

\section{Narratives}

These stories were generated from the students' individual experiences and crafted into an aspect of care that the students were most engaged with. The aspect of care became the focus of a more specific research question. The research questions were derived so that many methodological options could be applied to answer the question. This student cohort has chosen approaches that include formal systematic literature reviews, retrospective database audits, and geospatial analysis. Here are their stories.

\section{Vinh}

Who: Vinh is a 65-year-old father of two children. He grew up in Vietnam, then moved to Australia when he was 30 years old. Vinh started work in a factory, a physically demanding job that has helped to keep him fit throughout the years. However, he has recently retired and has let himself indulge in his favorite pastime: watching Vietnamese TV shows with a beer or wine. Now, his only form of physical activity is gentle aerobic stretches.

What: Vinh always craves the tastes of Vietnam-of home. His wife, who is also Vietnamese, often acquiesces and cooks traditional meals. Each dish has its own story or memory attached to it; for example, the fried floury fish patties dipped in salty fish sauce were what he grew up eating, and these must be paired with bowls of fluffy Jasmine rice because what else would you eat this with? His diet is also heavily influenced by his friends who are all Vietnamese. When he goes to his general practitioner (GP) for his annual checkup, the GP says that Vinh is pre-diabetic.

Aspect of care: Vinh's GP is Vietnamese, so the consultation occurs in Vietnamese, and there is no language barrier present. However, the lifestyle advice from his GP is brief: eat less stir-fried and fried foods, and exercise more until you break a sweat.

Research Question: How do cultural factors impact on health promotion in culturally and linguistically diverse patients with Type 2 diabetes?

\section{Oliver}

Who: Oliver is 28-years old and works full-time in the eastern suburbs of Sydney as a carpenter. Since he was young, Oliver has been in love with water sports and has been lucky enough to come from a family that has been able to support his love for surfing and the ocean. Despite working full-time, he spends a considerable amount of time in the sun most afternoons and on weekends surfing.

What: Last year-with considerable hesitancy-Oliver went for his first ever skin check, after being convinced by one of his friends it would be a good thing to do. What they found on Oliver's right shoulder took him by complete surprise. A small melanoma that had been mere millimetres away from metastasising.

Aspect of Care: So many emotions have filled Oliver since the excision of his melanoma. How had he, as a young, fit, and sun-smart 28-year-old, gotten a melanoma? Why did he wait until 28 to get his first skin check? Why isn't this issue talked about more among the surfing community? Sam is now more careful than most would feel is normal.

Research Question: Is there a correlation between melanoma incidence, severity, and stage to an individual's socio-economic status and geographical location: a geospatial analysis?

\section{Angela}

Who: Angela is a 25 -year-old woman who was recently married and is now looking to start her own family. Angela has suffered from severe anxiety for as long as she can remember. She was diagnosed when she was 18 and commenced on Fluoxetine at 19, which she has taken every day since. 
What: Angela is extremely worried about her likely antidepressant use during pregnancy and feels helpless due to the deficiency of alternatives. Fluoxetine is classed as a Category $\mathrm{C}$ drug in pregnancy, which means it has caused or is suspected of causing harmful effects on the human foetus or neonate. Current guidelines suggest that Fluoxetine is safe for use during pregnancy; however, some studies show an increased risk of foetal malformations and premature delivery are associated with selective serotonin reuptake inhibitor (SSRI) use in pregnancy.

Aspect of Care: The advice given to Angela by her GP regarding use of Fluoxetine if she was to fall pregnant was that it is not worth ceasing her medication if it posed a risk to her mental health. However, no other non-pharmacological alternatives were offered or discussed.

Research Question: Are there safe and effective alternative treatments for anxiety during pregnancy?

\begin{abstract}
Anna
Who: Anna is a 22-year-old busy and bubbly physiotherapy student who is motivated and determined to one day own her own sports physiotherapy clinic. Although she moved out of her family home several months ago, she is very close to her family, seeing them multiple times a week for dinners and other catch ups. She has lots of friends, however, she feels that she has drifted apart from a lot of them recently. Anna plays netball a couple of times a week and is generally fit and healthy. She moved to a different part of the city six months ago to be closer to work. She lives with her boyfriend, Peter, in an apartment. Anna has been in a relationship with her boyfriend for just over two years. Over the past year, they have been getting more serious: moving in together, talking about the future, planning when they want to get married, when they will have children, and in which suburb they might want to settle down in.
\end{abstract}

What: In the past six months Peter has been getting increasingly volatile both verbally and physically. He broke Anna's phone after a fight when he threw it against a wall. Their fights were escalating quickly and on a number of occasions she feared for her physical safety. Peter was becoming increasingly controlling, demanding to know what she had been doing, and who she was spending time with. He no longer wanted her to talk to her friends or family.

Aspect of Care: Anna presents to her GP with increasing fatigue over the last six months associated with anxiety. She is feeling socially withdrawn and isolated. Anna is reluctant to disclose her personal circumstances to her GP.

Research Question: What is the prognosis for women who experience intimate partner violence (IPV) and are unable to leave the relationship?

\title{
Ming and Zhong
}

Who: Zhong is 76 years old. He grew up in the city of Shanghai, China, where he worked as an academic at an esteemed university. In 1995, he moved to Australia with his wife Ming and their two children. When they arrived, they didn't speak any English and his university qualifications were not recognised. In order to provide for his family, Zhong took a job as a cleaner. A few years later Zhong and Ming opened their own restaurant in a suburb with a predominantly migrant Chinese population.

What: Zhong has been healthy all his life, however, over the last year, Ming and the children have noticed that he is becoming more forgetful. As Zhong's memory worsens, Ming has taken on more responsibility at the restaurant. They have also noticed that Zhong is having increasing difficulty with his English, struggling with words and phrases he previously used with relative ease. However, despite his cognitive decline, Ming has not mentioned his increasing forgetfulness at recent GP visits. There is significant stigma around this in Chinese culture, and it is often considered shameful. It is not until four years after she first notices symptoms that Ming finally addresses the issue with the GP. Zhong is diagnosed with dementia.

Aspect of Care: Ming is particularly reluctant to entrust Zhong's care to strangers. In Chinese culture it is expected that family members will continue to care for each other, so she continues to look after him at home. But as his condition progresses, she is not sure how much longer she can manage on her own. Ming herself has osteoarthritis, hypertension, 
and impaired vision. She has had no time lately to attend her own GP appointments. As a result, her health is also declining.

Research Question: While there is significant research on carer burden of those supporting people living with dementia, this is not the case for culturally and linguistically diverse (CALD) carers. What is the impact on the health of older carers of a CALD person living with dementia?

\section{Michael}

Who: Michael is a 23-year-old in his final year of his undergraduate degree at university. He is a healthy and active young man, having grown up playing rugby, basketball, and touch football. He is a very sociable character and loves his lifestyle.

What: Michael has no significant past medical history and rarely needed to visit his GP. But over a couple of weeks, Michael started to feel generally unwell, fatigued, and nauseous. He ignored these vague non-specific symptoms attributing them to being run down from university, which in the past had amounted to nothing and abated within a few days. A few months later, he noticed a small painless lump in his left testicle. Michael being the eldest child of three, felt too awkward about talking to younger family members for advice, so instead he left it for a few more weeks in the hope that it would spontaneously resolve. When the lump did not go away, Michael had to accept that something might be wrong and made an active decision to book an appointment with a GP.

Aspect of Care: At the first consultation Michael was told that "it was all fine and nothing to worry about". But this did little to clear Michael's mind and he left with a sinking feeling that something was simply "not right". After battling with these feelings for a few weeks he decided to alert his family into what has been happening and he was again booked into see a GP. This time, the GP suspected it was a varicocele and wrote up a non-urgent referral to a urologist. The next available appointment was in three months. The urologist sent him off for a scrotal ultrasound. Again, the next available scan was a few days later. Upon review of the ultrasound, the urologist diagnosed Michael with testicular cancer and urgently referred him onto the oncologist. It was December 23 of that year when he was finally diagnosed. Michael went in for orchiectomy surgery the following day, Christmas Eve. This was eight months after he initially noticed the lump and seven months after he first presented to a health professional.

Research Question: What factors lead to delays in diagnosis of testicular cancer in young males?

\section{Julie and Daniel}

Who: Julie, Daniel, and their two children were a typical working-class Australian family. Julie and Daniel were brought together by their passion for photography and outdoor adventures. They eventually married, had a family, bought a house, and enjoyed many family holidays. Like most families, things weren't always perfect, and Julie and Daniel also had their share of arguments and disagreements, financial difficulties, and work stresses. In spite of the difficult times, they loved each other and were quite satisfied with their lives.

What: About four years ago, Julie and Daniel's life was forever changed after Daniel was diagnosed with a terminal illness. Daniel was given a 10 -year prognosis. The doctors said that there was no available treatment for this rare condition and virtually no chance of remission. Daniel and Julie now live in the hope that a new treatment will become available. In the meantime, Daniel's health is gradually declining, his moods unpredictable or sullen, and Julie finds herself managing the roles of a worker, career, wife, and mother.

Aspect of Care: Four years after Daniel's diagnosis, Julie experiences what could be described as an emotional breakdown; she becomes unable to cope with everyday life and requires long-term sick leave and emergency mental health support.

Research Question: What is the impact of a terminal illness diagnosis on the physical health of the caregiving spouse? 


\section{James}

Who: James is a 22-year-old carpenter, with strong ambitions to develop his own building and project development business. He works incredibly hard, six days a week, 50 weeks of the year. He bought his first a house at 21. James is the only son of four children. When he has time off, he helps his father on the family property, building sheds and fixing fences. James plays sport and enjoys being active with his mates.

What: Recently, James has been having issues with his lower back at work. As one of the youngest onsite, he is often given physically demanding roles such as shovelling dirt or moving timber, instead of technically difficult and less physical roles. Coming from a rowing background at school, James is used to "putting up with" a sore back.

Aspect of Care: James feels the pressure of being the youngest onsite, part of a team that is trying to meet a deadline. If he complains about having a sore back, he is ridiculed for being "soft", and often told to get on with the job. When he gets home from work, he self-treats the issue with heat and topical ibuprofen gel, exhausted and in need of rest for the next day's work. Work-related social pressures have the potential to influence work practice, heightening the risk of physical and mental harm.

Research Question: What is the influence of workplace culture on the incidence of low back pain in workplaces with high physical demand?

\section{Elizabeth}

Who: Elizabeth is a 30-year-old female accountant, living in a Sydney apartment. Her workday begins with a 40-minute commute on the train, followed by a 9-hour workday, with approximately 2-3 hours overtime per week.

What: Elizabeth often finds herself working through lunch and snacking during the day to keep her energy levels up. When she does have a lunch break, Elizabeth often attends one of the many lunch meetings where food is plentiful and calorie dense. The last 10 years has seen her waistline grow and her weight increase from $60 \mathrm{~kg}$ to $100 \mathrm{~kg}$. This weight gain is mirrored by her husband of five years, Phillip, who has been overweight since his mid-twenties. A nonsmoker and social drinker, Elizabeth tries to eat well during the week but barriers, such as being time poor, prevent her from adequate meal preparation and instead, see her reach for takeaways or frozen foods. Exhausted by work and life in general, Elizabeth perceives the thought of going to the gym as impossible.

Aspect of care: Elizabeth attends her local GP to discuss bariatric surgery, to help her lose weight. On the day of her appointment Elizabeth has a BMI of 35, no diagnosed co-morbidities, no medical conditions, no medications, no allergies, and no significant family history. She is tired of trying "everything" without result and sees weight loss surgery as the easy route to a long-term solution. Elizabeth is not interested in hearing about possible complications. She just wants to stop struggling with her weight and regain her confidence.

Research Question: What is the prevalence of long-term complications over the lifespan of a person following weight-loss surgery?

\section{Andy}

Who: Andy is a 15-year-old passionate soccer player from the North West suburbs of Sydney. He is balancing training for his school and local club teams during the week and scrambles to fit in two games of soccer on the weekend. He is a well-rounded sportsman and has not suffered any major injuries to date. Previously he has suffered from a few scratches and contusions as well as a minor ankle strain last year that resolved within a couple of days. He believes that he is on track to be selected in the 1st grade opens squads for both of his current teams by next year and has recently increased the intensity of his training.

What: During a warm-up drill on Saturday morning, a teammate attempts to tackle the ball in front of Andy, missing the ball and kicking Andy's knee. In doing so, the team mate inflicts a medially directed force on the outside of Andy's knee, damaging his medial collateral ligament. Andy falls to the ground in pain, clutching his right knee. He is helped up by his team mates and hobbles over to the sideline, struggling to weight bear on the right side. His coach retrieves him some ice and tells him to lie on his back and elevate his knee. 
Aspect of Care: Andy is concerned, "How serious is it? What should I do? How long will I be out? Can I do anything to speed up the recovery?" His coach struggles to answer his questions, and asks Andy's mother to take him up to the local GP. Andy's mother reassures him that she doesn't think it is too serious and that her good friend is a physiotherapist. She wonders if the doctor will recommend seeing her to assist with returning back to playing soccer as soon as possible.

Research Question: What factors determine whether GPs will refer patients with acute soft tissue knee injuries to physiotherapy?

\section{Sally}

Who: Sally is a 48-year-old mother to two young adults. She had a very close relationship with her mother and in her early adult years she was a very warm, social, organised person.

What: Sally struggled with the gradual loss of her mother to dementia and turned to alcohol as a means of coping. Sally has since divorced her husband, become estranged from her children, and spends most of her days alone drinking in various pubs near her accommodation. She is unemployed and recently sold her bed in a bid to buy more alcohol.

Aspect of Care: Sally has had multiple unsuccessful attempts at rehabilitation. In her latest effort she stayed voluntarily for six months only to relapse on the journey home. Since her return from rehabilitation, Sally has gone missing on multiple occasions putting immense pressure on what remains of her family to locate and pick up the pieces. She is often found in local motels, gaunt, with bruises on her arms and face. The doctors at the Alcohol and Drug Dependency Centre told her family that Sally requires a 12-month involuntary stay at a rehabilitation centre, however, the only available program is 21 days. Sally is both the "heart sink" patient and mother, gripped by an illness that her family fear she will never overcome.

Research Question: What is the impact on doctors when faced with the challenges of managing patients with long-term illness related to personal choice?

\section{George}

Who: George didn't go to the doctor or hospital much as a child. His mother was a nurse, and when he was unwell or hurt she often didn't think it was serious enough to warrant a visit to a doctor; "See how you feel tomorrow" was a constant refrain. As George got older, his mother spoke more often about the hassles and inconveniences of the health system, and how this deterred her from going to the doctor herself and from taking her children. She also regularly spoke of clogged emergency departments and GP clinics that were filled with people who would just get better on their own.

What: As George got older he found he held similar views to his mother. He had long felt that attending hospital or a GP clinic was mainly an inconvenience and would tolerate feeling quite unwell to avoid it, even though he had no justification for this feeling. George believed that most of the time things get better if you just wait. George didn't stop to think why that was the case, he just believed it was. George had never been seriously unwell, so his experience of being sick had always been to feel better fairly quickly and often without the help of a healthcare professional.

Aspect of Care: Now as an adult George feels very comfortable attending a doctor and sees the value in doing so, but generally won't unless he thinks it's an issue that won't resolve without intervention. He will regularly attend work unwell because he feels confident that it will pass and doesn't want to go through the hassle of arranging a doctor's appointment for something that will resolve on its own and won't require any active treatment.

Research Question: What is the help-seeking behavior of children of health professionals? 


\section{Jack}

Who: Jack is a 40-five-year-old construction worker, husband, and father of two young boys. Jack has been working in construction since he was 16 .

What: Two years ago, Jack injured his back while attempting to drag a pallet off the back of his van by himself.

Aspect of Care: Since the injury, Jack has been managing his pain with over-the-counter (OTC) codeine products, such as Panadeine and Nurofen Plus. However, on February 1, 2018, a nationwide ban on OTC sales of medications containing codeine was instated. Since then, Jack has been unable to obtain codeine-containing medication, as his regular GP does not feel prescribing a drug with a high potential for addiction is suitable for chronic back pain. In the meantime, Jack says he has been drinking "a few more cans of beer at night to help take the edge off, doc". He says that it provides him with enough relief to work through the pain, and that taking time off from work isn't an option as "money is short at the moment". Jack asks his GP in a drop-in medical centre if she would be willing to write him a prescription for codeine-containing medication.

Research question: What is the impact of the over-the-counter ban of codeine-containing medications on patient outcomes and prescription rates?

\section{Jane}

Who: Jane is 42-years-old and lives at home with her husband and three children (5, 11, and 13 years old). Her husband is an accountant at a mid-tier firm, and they live in a modest home in a leafy neighbourhood on Sydney's lower north shore. Jane was working as an account director at a consultancy company before she had the kids. She intends to return to work once the youngest one starts school, but it's looking increasingly impossible with her husband's recent promotion and the poor affordability of after school care options.

What: After Jane turned 18, like many of her friends, she drank in a classic binge-drinking pattern. This changed as she moved into full-time work where the possibility of networking associates and clients necessitated her attendance at lunch meetings and Friday night drinks. As she moved up the company, the increased responsibility saw her leaning on alcohol to help her sleep, and she'd frequently find herself having two glasses of wine with dinner. She avoided alcohol throughout her pregnancies, but now that the kids are a little older, alcohol is again a big part of life. Jane typically drinks a bottle of wine a night. She will have her first glass while preparing dinner, and by the end of the night the bottle will be empty. Sometimes it's two bottles. Jane will often attempt to conceal her drinking from her husband and kids by placing bottles in the neighbours' recycling bins. The eldest of her children has started to joke about it as if it's a normal part of life "it's wine o'clock, that means dinner is soon", but he doesn't like how grumpy mum is in the morning. Despite her drinking, she wakes up the next morning before anyone else in the house, does the washing and makes all the lunches. She is for all intents and purposes the doting mother and wife. The grogginess dissipates at some point late in the morning, however, by $4 \mathrm{pm}$ she is checking the clock to see if it's appropriate to pour her first glass.

Aspect of Care: Jane is self-conscious about how much weight she has gained over the last couple of years. She used to pride herself in her ability to drop the weight after the pregnancies, but after her last child was born, the weight hasn't budged. Jane's BMI is now 28. It doesn't help that she's often too hungover to exercise.

Research Question: What is the prevalence of occult alcoholism in Australian females aged 35-59 years old?

\section{John}

Who: John is 21 years old and a full-time university student. His studies continually keep him busy with long hours and minimal sleep. To alleviate his stress and fatigue, John often indulges in eating sweets and drinking multiple coffees. Alongside his studies, John also juggles a part-time teaching job, sporting commitments, and volunteering responsibilities. He is an avid sports player, regularly playing in tennis and basketball competitions. As a social butterfly, he also has a love for adventure and enjoys travelling and exploring the world. 
What: As a Type 1 diabetic, John's activities often disrupt his BGL control and contributes to a perpetual cycle of increasing stress and poor overall physical and mental health.

Aspect of Care: Despite his love of sport, John often feels frustrated at the impact his diabetes can have on his performance, despite his best efforts to control his BGLs. His family and friends are a major source of support and keep him positive about dealing with his diabetes. John regularly seeks advice from his endocrinologist who carefully examines his "numbers" and provides appropriate advice about his medications and diet. However, they quickly dismiss his deeper concerns that his diabetes is negatively impacting on his choices.

Research Question: Can we achieve therapeutic targets for people with Type I diabetes with reference to their lifestyle choices? 